Planetary Systems in the Universe - Observation, Formation and Evolution

Proceedings IAU Symposium No. 202, (C)2004 IAU

Alan Penny, Pawel Artymowicz, Anne-Marie Lagrange, \& Sara Russell, eds.

\title{
Results from Microlensing Searches for Extrasolar Planets
}

\author{
Penny D. Sackett \\ Kapteyn Institute, 9700 AV Groningen, The Netherlands
}

\begin{abstract}
Specially-designed microlensing searches, some of which have been underway for several years, are sensitive to extrasolar planets orbiting the most common stars in our Galaxy. Microlensing is particularly well-suited to the detection of Jupiter-mass planets orbiting their parent stars at several AU. Since Jovian analogs are thought to influence the subsequent evolution of most planetary systems, they are particularly important to study. The orbital radii and distances to the planetary systems probed by microlensing are larger than those currently studied by radial velocity techniques; the two methods are thus complementary. Recent results from microlensing searches are discussed, including constraints on Jovian analogs orbiting typical Galactic stars. Benefits and drawbacks of the technique for the characterization of planetary systems, and future prospects are briefly reviewed.
\end{abstract}

\section{Introduction}

The first detection of an extrasolar planet around a normal star (Mayor \& Queloz 1995) and subsequent deluge of similar discoveries by the radial velocity technique (Marcy, Cochran \& Mayor 2000), have taught us that $~ 5 \%$ of solar type stars harbor planetary systems very unlike our own. What remains to be determined is the abundance of planetary systems similar to that of the Sun's (eg., terrestrial planets at $\sim 0.5-2 \mathrm{AU}$ or Jovian analogs at $\sim 3-12 \mathrm{AU}$ ) and the frequency of planets around the most common stars in our Galaxy, M dwarfs. Microlensing is providing the first partial answers to these questions.

\section{Galactic Planetary Systems As Multiple Microlenses}

Microlensing occurs whenever a massive compact object (such as a star, black hole, etc.) passes very near the line-of-sight to a background luminous source. In the case of a single point lens, two images of the source are formed with a separation that scales with the angular radius of the Einstein ring, $\theta_{\mathrm{E}} \equiv$ $\left[4 G M(1-x) /\left(c^{2} D_{L}\right)\right]^{-1 / 2}$, where $x \equiv D_{L} / D_{S}$, and $M, D_{L}$, and $D_{S}$ are the mass of the lens, its distance, and the distance to the source, respectively. For sources in the Galactic bulge, this separation is $\sim 1$ mas, and thus generally too close to be resolved. The combined image brightness, however, can be observed and is a function of the changing projected distance between the lens and the observer-source line-of-sight. As the source moves through the axisymmetric magnification pattern generated by a single lens, a symmetric light curve results.

This symmetry is destroyed for multiple lenses such as binary star systems or planetary systems. More complicated magnification patterns are formed, and 


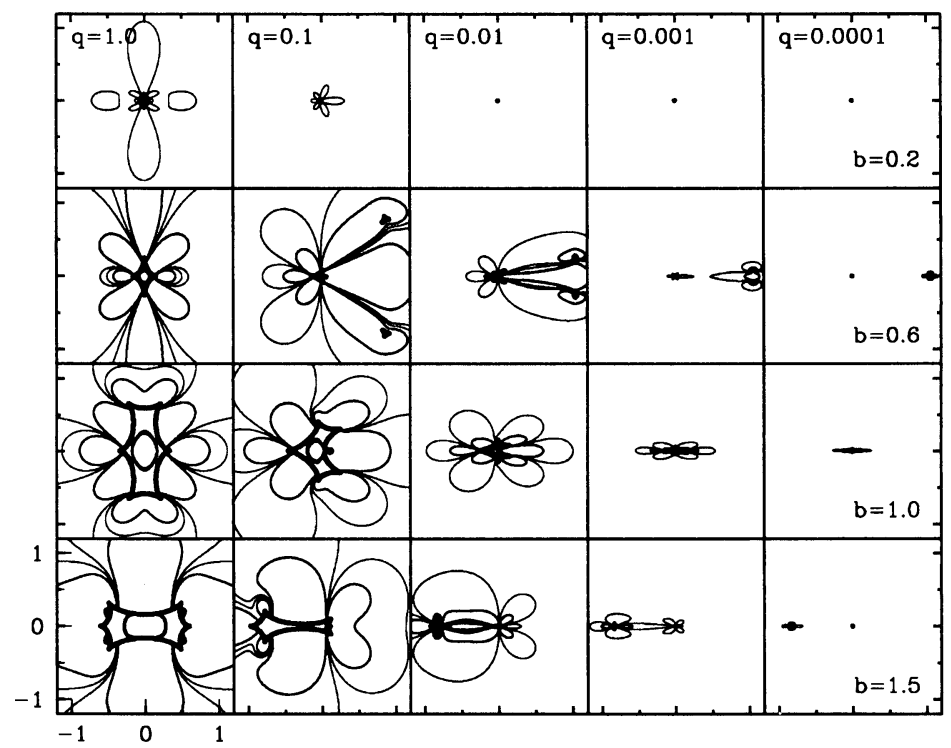

Figure 1. Magnification patterns for binary lenses with mass ratio $q$ and projected separation $d$ ( $=b$ in this figure). Differences in magnification of $\pm 1 \%$ and $\pm 5 \%$ between a point lens and a binary of the same total mass are shown as contours. The heaviest contours are the "caustic curves." A Jupiter-Sun lensing system would have $q \approx 10^{-3}$ and $d \approx 1.2$ if located halfway between the observer and the Galactic bulge and viewed face-on. (Adapted from Gaudi \& Sackett 2000).

the resulting light curve depends on the angle $\phi$ of the source trajectory. For binary lenses, the topology of the magnification map (Fig. 1) depends on only two parameters: the mass ratio $q \equiv m_{1} / m_{2}$ of the lenses and their projected separation $d \equiv a_{p} / R_{\mathrm{E}}=a_{p} /\left(D_{L} \theta_{\mathrm{E}}\right)$ in units of the Einstein ring radius. For typical Galactic lenses, $M$ dwarfs in the bulge or inner disk, the physical size of the Einstein radius is $R_{\mathrm{E}} \sim 2-3 \mathrm{AU}$. Because typical $R_{E}$ are comparable to the orbital radius of many Solar System planets, microlensing is an excellent way to search for planets, as first suggested by Mao \& Paczynśki (1991) and further developed by Gould \& Loeb (1992).

Figure 1 shows the differential magnification effect of binary lenses as a function of their mass ratio $q$ and separation $d$. Each panel covers an area $\sim \theta_{\mathrm{E}}$ on a side centered on the more massive primary lens. The brightness of an image is proportional to $|\operatorname{det} J|^{-1}$, where $J$ is the Jacobian that describes the coordinate transformation between the image and source planes. The locus of points in the source plane for which $|\operatorname{det} J|=0$ is called the "caustic curve." A source crossing the caustic will be highly magnified.

Since most microlensing events are alerted in real time only when the source lies near or inside $\theta_{\mathrm{E}}$, a light curve will reveal the binarity of the lens (via an anomaly $>1 \%$ ) only if the source trajectory passes over the contours shown in Fig 1. Otherwise, the light curve will be indistinguishable from that due to a single isolated lens. Figure 2 illustrates this effect; two possible source trajectories are shown passing the same distance from the "central" caustic, but 

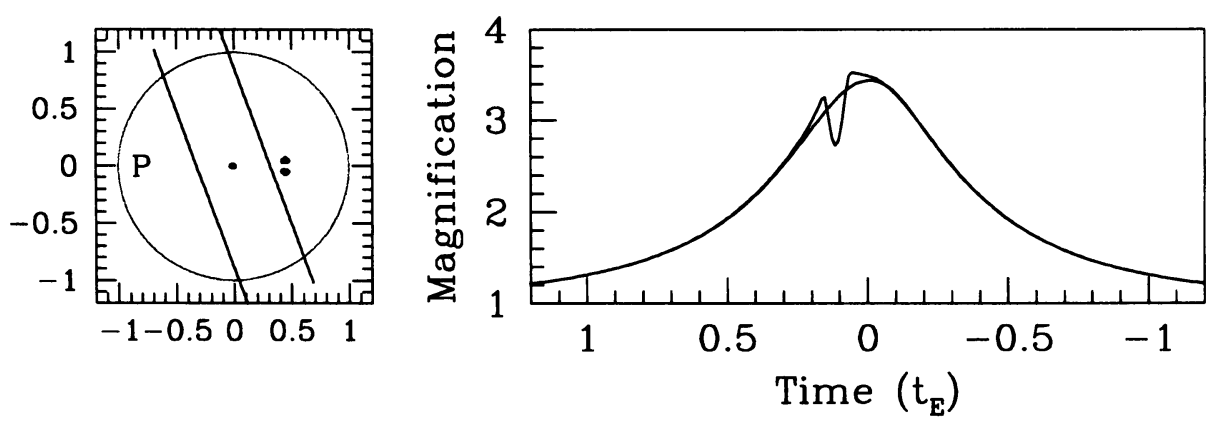

Figure 2. Left: The position of a $q=10^{-3}$ planet is marked with "P" inside the Einstein ring radius of its primary at $d=0.8$. The central caustic and two "planetary caustics" are shown, along with two possible source trajectories. Note that the caustics do not coincide with the position of planet. Right: The resulting light curves.

different distances from the two, outer "planetary" caustics (all three caustics are caused by the lens-planet combination). The light curve associated with only one of these trajectories betrays the presence of the planet. Since the deviation contours are most extended for $q$ and $d$ of order unity (Fig. 1), these types of binary lenses will be the easiest to detect.

\subsection{Sensitivities vs Efficiencies}

Even planets as small as the Earth generate caustic structures as they orbit Galactic lenses; background sources passing over or near these caustics will experience a substantial additional microlensing effect due to the binarity of the lens. The cross section of the planetary caustic and thus the probability that a random trajectory will pass over or near it decreases slowly with mass ratio (Dominik 1999). For this reason, microlensing - unlike most other planet detection methods - has sensitivity to terrestrial-mass planets. This sensitivity is limited primarily by the sampling rate and precision of the photometry required to see signals that are reduced in amplitude by finite source effects for $q \leq 10^{-4}$. The efficiency with which planets of given $q$ and $d$ can be detected in a given light curve is a statistical quantity that depends on the photometric precision, sampling and duration of the photometric data and on the characteristics of the underlying event sampled, most notably its minimum impact parameter, $u_{o}$, and the amount of blended non-lensed light in the same resolution element (Gaudi \& Sackett 2000). Determination of detection efficiencies for individual light curves allows non-detections to be translated into statistical upper limits for a given class of planet.

\subsection{Detection vs Characterization}

In principle, the detection of a microlensing anomaly can be quantified statistically, but its clear identification with a planetary lens relies on the characterization of the planet through determination of $q$ and $d$. Since any given event will not repeat, characterization must be obtained at the time of detection, and 

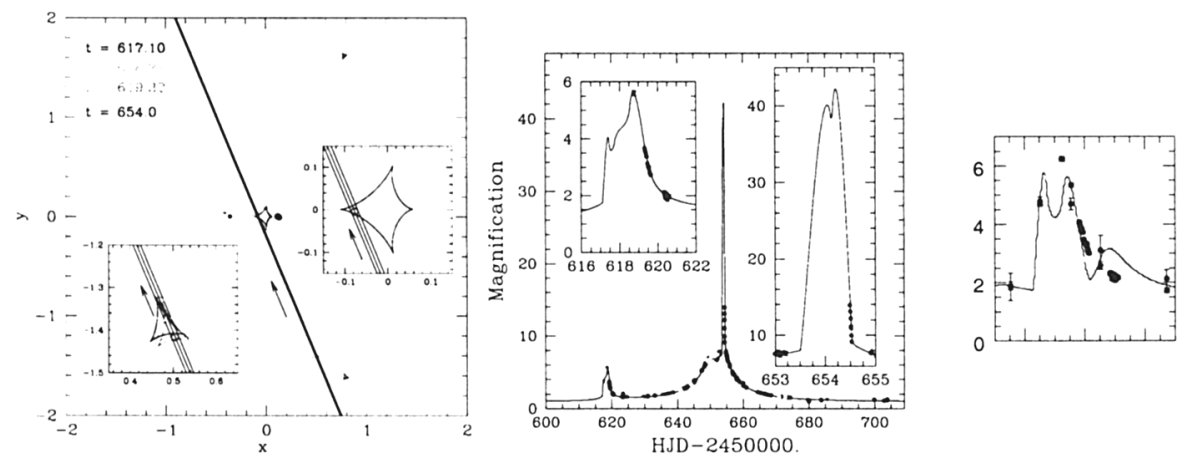

Figure 3. Left: Moving caustic structure of rotating binary model of MACHO 1997-BLG-41. Inserts show zooms; arrow indicates source motion. Middle: Rotating binary fit to multi-site PLANET team light curve. Right: Static binary+planet model atop PLANET (red) and MACHO-GMAN (black) data over first caustic feature $(\S 3.1)$.

will require better data. With sufficient data quality, both $d$ and $q$ can be determined from light curve modeling, although in some cases the well-known $d-d^{-1}$ "degeneracy" (Griest \& Safizadeh 1998; Dominik 1999) will prevent a unique identification. High magnification events are sensitive and reasonably efficient to the detection of small mass planets since their small $u_{o}$ brings them close to the central caustic generated by companions (Griest \& Safizadeh 1998). Unfortunately, such detections may be particularly difficult to characterize, since all planets orbiting the primary lens will distort the central caustic (Gaudi, Naber \& Sackett 1998); more massive ones will have an effect over a larger range of $d$. Di Stefano and Scalzo (1999) point out that current programs typically halt intensive monitoring when $u \geq 1$; extended high-precision monitoring may detect and characterize planets at larger separation from their host lenses.

\section{Have Exoplanets been Discovered with Microlensing?}

Of 21 light curves consistent with binary lenses analyzed by MACHO, two produced acceptable fits with companion masses as small $0.05 M_{\odot}$ (Alcock et al. 2000). The first suggestion of possible planetary-mass lenses (Bennett et al. 1997) came from MACHO survey data for MACHO 1994-BLG-4, which could be modelled as an M-dwarf/5 $M_{J}$ pair, and MACHO 1995-BLG-3, a very short duration event modelled as an isolated $2 M_{J}$ lens. However, many alternate interpretations were allowed by the infrequently sampled light curves inherent to survey data (which must sample $\sim 10^{6}$ stars nightly!); no firm planetary detection was claimed in either case.

The timescale $t_{\mathrm{E}}=\theta_{\mathrm{E}} / \mu$ ( $\mu$ is the relative proper motion of lens and source) of typical Galactic microlensing events is weeks to months; whereas planetary $(q \leq 0.01)$ anomalies would have durations of hours to days. The necessity of round-the-clock monitoring for detection and characterization of short-lived planetary deviations prompted the establishment of international collaborations 


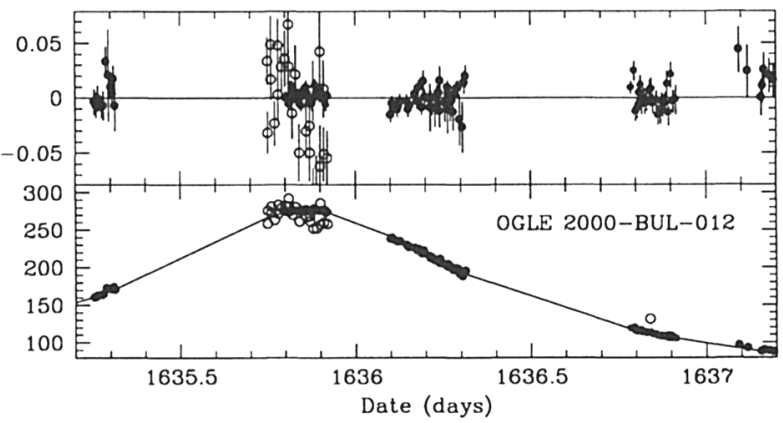

Figure 4. Bottom: Single-lens fit to OGLE 2000-BUL-12 light curve data. Flux units are linear and arbitrary. Black, open circles are OGLE data; colored, filled dots are multi-site PLANET data. Top: Residuals from the single-lens model expressed as a fraction of the flux.

such as MPS (Rhie et al. 2000) and PLANET (Albrow et al. 1998), which obtain sub-day to sub-hourly photometry on events discovered by the survey teams MACHO, OGLE and EROS. Although tantalizing hints have been seen in some light curves (see below), no clear planetary anomaly has been detected.

MACHO 1997-BLG-41: The very unusual event MACHO 1997-BLG41 caused a stir in the community: although clearly multiple lens microlensing, static stellar binary models did not fit the data. Bennett et al. (1999) interpreted the double caustic structure as coming from a static triple system, a Jovian planet orbiting a stellar binary. The PLANET team, modeling their own data, instead showed that the light curve could be fit as a normal stellar binary (Albrow et al. 2000a) whose rotation brings one of the triangular caustics across the source trajectory (Fig. 3). The proposed binary+Jovian model was incompatible with PLANET data.

OGLE 2000-BUL-12: Recently, Yock et al. (2000) have suggested that a planet may be orbiting the primary lens of event OGLE 2000-BUL-12, based on analysis of public domain OGLE data (Udalski \& Szymanski 2000) that displayed a possible anomaly at peak. Unpublished PLANET team data indicates no anomaly and much smaller scatter at the same temporal position (Fig. 4).

MACHO 1998-BLG-35: Although ruling out a large class of high-mass planets orbiting the lens of MACHO 1998-BLG-35 ( $\$ 4.1$ ), the MPS and MOA groups (Rhie et al. 2000) noticed a weak anomaly near the peak of this high amplification event that they interpreted as intriguing evidence of a low-mass companion with $4 \times 10^{-5}<q<2 \times 10^{-4}$. The signal fell below the formal MPS/MOA detection limit. PLANET team data for this event did not confirm this detection; the PLANET light curve was consistent with an isolated point lens (Albrow et al. 2000c).

\section{Limits on the Abundance of Jovian Planets in the Galaxy}

Current microlensing planet searches have appreciable efficiency for the detection of companions with mass ratio $q \geq 10^{-4}$, ie., planets with masses of order 

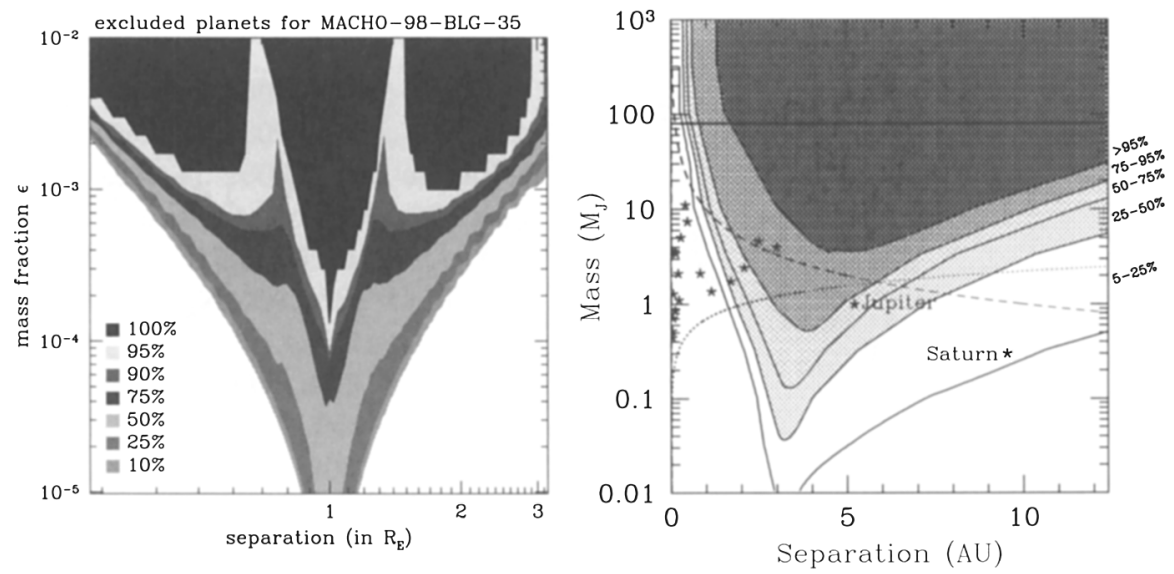

Figure 5. Left: Planetary exclusion contours at various significance levels as a function of mass fraction and $d$ for companions to event MACHO 98-BLG35 (Rhie et al. 2000). Right: Same for event OGLE 1998-BUL-14, where the planetary mass and orbital separation have been estimated assuming a $1 \mathrm{M}_{\odot}$ mass lens with $R_{\mathrm{E}}=3 \mathrm{AU}$ (Albrow et al. 2000b). Asterisks mark known radial velocity planets.

$m_{p} \geq 0.1 M_{J}$. The lack of detected perturbations consistent with planets in this mass range allows constraints to be placed on the abundance of Jovian analogs around typical stars (ie, lenses) in the Milky Way.

The first published exclusion diagram (Fig. 5) for companions in an individual lensing system was presented by the MPS/MOA collaborations for MACHO 98-BLG-35 (Rhie et al. 2000). Due to the high amplification of this event, the source would have passed very near any (central) caustic structure due to massive companions. Since no anomaly consistent with Jovian-mass companions was seen, exclusion contours could be derived as a function of $q$ and $d$ for this lensing system (Fig. 5). The PLANET collaboration performed a similar analysis for another high amplification event, OGLE 1998-BUL-14 (Albrow et al. 1999b). When converted to parameters appropriate to a solar-type lens in the bulge and averaged over all orbital inclinations, the exclusion contours for Jovian and super-Jovian planets in the OGLE 1998-BUL-14 overlap the region of parameter space inhabited by current planet detections by the radial velocity technique (Fig. 5). For the most part, however, microlensing is sensitive to planets at larger orbital radii (1-10 AU) than is the Doppler technique.

Based on 43 microlensing events collected from five years of photometric observations, the PLANET team has recently announced constraints on the abundance of Jovian and super-Jovian planets orbiting typical stars (ie., lenses) in the Galaxy (Gaudi 2000, Albrow et al. 2000c). No clear planetary anomalies were observed in these 43 events, implying that less than one-third of $\sim 0.3 \mathrm{M}_{\odot}$ stars have Jovian-mass companions with semi-major axes in the range $1.5 \mathrm{AU}<$ $a<4$ AU (Fig. 6). Weaker limits are placed on the existence of Jovian planets orbiting in the range $1 \mathrm{AU}<a<7 \mathrm{AU}$. These are the first constraints on exoplanets orbiting the most common of Galactic stars: M-dwarfs. 

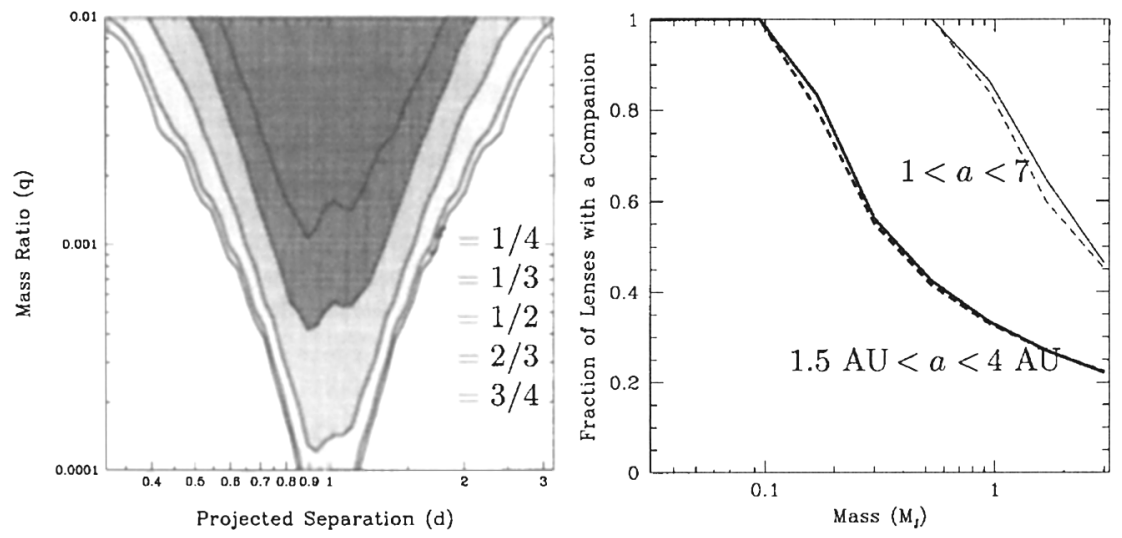

Figure 6. Left: Upper limits (95\% significance) on the presence of companions in a given fraction $f$ of microlenses, shown as contours in mass ratio $(q)$ - projected separation $(d)$ space. Statistically, $q=0.001 \rightarrow m_{P} \approx 0.3 \mathrm{M}_{\text {Jup }}$ and $d=1 \rightarrow r_{P} \approx 2 \mathrm{AU}$. Right: Upper limits on $f$ as a function of companion mass for orbital radii in the indicated ranges (adapted from Albrow et al. $2000 \mathrm{c}$ ). The negligible effect of finite source size on the efficiency calculations is also shown (dashed).

\section{Prospects for the Future}

As with all techniques, microlensing has advantages and disadvantages. In the cadre of exoplanet search techniques, it offers the opportunity to detect and characterize Jovian planets at large orbital radius (1-10 AU) without waiting for one or more orbital periods. Currently, it also has more sensitivity to Neptune-mass planets than any other ground-based search technique. In the future, this sensitivity may be enhanced and extended with new facilities on the ground (Peale 1997, Sackett 1997) and in space (Rhie, this conference). Several challenges must be overcome to detect and characterize Earth-mass planets with reasonable efficiency, including the diluting effect of finite source size on the magnification gradient due to small caustics (Bennett \& Rhie 1996, Gaudi \& Sackett 2000).

Currently, the mass ratio (but not the mass) and the projected separation in units of $\theta_{\mathrm{E}}$ (but not in $\mathrm{AU}$ ) of a lensing planet can be determined from an anomaly. More information may be forthcoming in the future. Large-aperture spectroscopy of an event may yield spectroscopic identification of the faint primary lens against the sea of source star light (Mao, Reetz \& Lennon 1998), thereby allowing the mass of the primary and $\theta_{\mathrm{E}}$ to be estimated. Measurement of the photometric centroid motion due to the changing position and brightness of the microimages (astrometric microlensing), could yield the mass of the lens (Dominik \& Sahu 2000, Han 2000, Gould \& Han 2000), and a more robust determination of planetary parameters (Safizadeh, Dalal \& Griest 1999).

Acknowledgments. I am grateful to my PLANET colleagues for permission to display unpublished data for OGLE 2000-BUL-12. 


\section{References}

Albrow, M.D. et al. 2000a, ApJ, 534, 894

Albrow, M.D. et al. 2000b, ApJ, 535, 176

Albrow, M.D. et al. 2000c, ApJ, submitted (astro-ph/0008078)

Alcock, C. et al. 2000, ApJ, 541, 270

Bennett, D.P., \& Rhie, S.H. 1996, ApJ, 472, 660

Bennett, D.P. et al. 1997, ASP Conf. Prof. 119, 95 (astro-ph/9612208)

Bennett, D.P. et al. 1999, Nature, 402, 57

Di Stefano, R., \& Scalzo 1999, ApJ, 512, 579

Dominik, M., 1999, A\&A, 349, 108

Dominik, M., \& Sahu, K. 2000, ApJ, 534, 213

Gaudi, B.S. 2000, PhD Thesis, The Ohio State University

Gaudi, B.S., Naber, \& Sackett, P. 1998, ApJ, 502L, 33

Gaudi, B.S., \& Sackett, P.D. 2000, ApJ, 528, 56

Gould, A., \& Han, C. 2000, ApJ, 538, 653

Gould, A., \& Loeb, A. 1992, ApJ, 396, 104

Griest, K., \& Safizadeh, N. 1998, ApJ, 500, 37

Han, C., 2000, in A New Era of Microlensing Astrophysics, ASP Conf. Series, eds. Menzies \& Sackett, in press (astro-ph/0003369)

Marcy, G., Cochran, W.D., \& Mayor, M. 2000, in Protostars and Planets IV, eds. Mannings, Boss \& Russell (U. of Arizona Press: Tucson) p. 1285

Mayor, M., \& Queloz, D. 1995, Nature, 378, 355

Mao, S., \& Paczynśki, B. 1991, ApJ, 374, 37

Mao, S., Reetz, J., \& Lennon, D.J. 1998, A\&A, 338, 56

Peale, S. 1997, Icarus, 127, 269

Rhie, S.H. et al. 2000, ApJ, 533, 378

Sackett, P.D. 1997, ESO Document: SPG-VLTI-97/002 (astro-ph/9709269)

Safizadeh, N., Dalal, N., \& Griest, K. 1999, ApJ, 522, 512

Udalski, A., \& Szymanski, M. 2000, http://www.astrouw.edu.pl/ftp/ogle/ogle2/ews/ews.html 